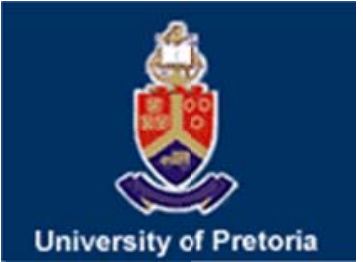

University of Pretoria Department of Economics Working Paper Series

Measuring Co-Dependencies of Economic Policy Uncertainty in Latin American Countries using Vine Copulas

Semih Emre Çekin

Turkish-German University

Ashis Kumar Pradhan

Rajagiri Valley Campus

Aviral Kumar Tiwari

Montpellier Business School and IFHE University

Rangan Gupta

University of Pretoria

Working Paper: 2018-67

October 2018

Department of Economics

University of Pretoria

0002, Pretoria

South Africa

Tel: +27 124202413 


\title{
MEASURING CO-DEPENDENCIES OF ECONOMIC POLICY UNCERTAINTY IN LATIN AMERICAN COUNTRIES USING VINE COPULAS
}

Semih Emre Çekin*, Ashis Kumar Pradhan ${ }^{\dagger}$, Aviral Kumar Tiwari ${ }^{\ddagger} \&$ Rangan Guptađ

Sept $2018-v 1.0$

\begin{abstract}
We analyze the dependence structure of economic policy uncertainty in four Latin American economies (Brazil, Chile, Colombia, Mexico) using vine copula modeling with various forms of tail dependence. Our results suggest that there are significant dependencies in economic uncertainty among the economies considered. We also find that tail dependence is more prevalent in the period preceding the Global Financial Crisis and becomes less relevant in the post-crisis period. Previous works suggest that uncertainty in economic activity can have substantial effects on economic issues ranging from business cycles to contagion effects of financial crises. Due to this relevance, our results have significant implications on the analysis of a range of macroeconomic issues, especially for emerging economies.
\end{abstract}

\section{JEL Classifications: .}

Keywords: Economic policy uncertainty, vine copula, emerging economies

*Department of Economics, Turkish-German University, Istanbul, Turkey.

${ }^{\dagger}$ Rajagiri Business School, Rajagiri Valley Campus, Kochi, India

${ }^{\ddagger}$ CESD, Montpellier Business School, Montpellier, France

$\S$ Department of Economics, IBS-Hyderabad, IFHE University, Hyderabad, India

『 Department of Economics, University of Pretoria, Pretoria, 0002, South Africa. 


\section{Introduction}

For many years emerging market economies have been subject to large swings in business cycles, financial market returns and macroeconomic fundamentals. During the years 19982002, most Latin American countries experienced economic turmoil and sluggish growth rates. Following this period, the years 2003-2007 marked a period of remarkable growth and stability, mainly due to favorable commodity prices and credit conditions, international trade, and remittances. However, when the global financial crisis (henceforth GFC) hit markets, Latin American countries were among the hardest hit in the emerging world in terms of sluggish growth and per capita GDP as compared to the boom years (Stiglitz (2010); Ocampo (2009)). Countries such as Colombia and Mexico witnessed the slowdown during the first half of the 2008, whereas, in other countries, especially Brazil, growth came to a halt in September 2008. Although measures taken by the Chilean authorities such as auctioning foreign currency denominated deposits to national banks, swap lines, and the flexibilization of reserve requirements helped to preserve stable conditions in the domestic economy (Chan-Lau (2010)) in the initial phase of the crisis, the Chilean economy experienced similar sluggish growth during 2009.

While many explanations are provided for the experience of these countries, a large body of research establishes that uncertainty shocks can influence economic fundamentals such as output, inflation and the interest rate. Especially after the effects of the GFC spilled over from the US to other economies, there was a surge in research related to policy uncertainty and spillovers. After the influential analysis of Bloom (2009), in which the author lays out how uncertainty shocks affect economic conditions, Colombo (2013) establishes in a structural VAR setup that uncertainty shocks originating from the US affect output and prices in the EU. The author also argues that US uncertainty shocks affect EU fundamentals stronger than EU uncertainty shocks. In a similar analysis by Klößner $\mathscr{E}$ Sekkel (2014), the authors first construct a policy uncertainty spillover index and then provide evidence that a significant portion of policy uncertainty countries emanates from other countries. In a more recent work, Biljanovska et al. (2017) show that uncertainty shocks from other countries can adversely affect economic fundamentals and that uncertainty spillovers from abroad account for the majority of the effect.

For emerging economies, previous work such as Fernández-Villaverde et al. (2011) and Carrière-Swallow $\&$ Céspedes (2013) argue that volatility and uncertainty surrounding real interest rates and global conditions can also have adversely effects on fundamentals.

These works typically utilize methods such as DSGE or VAR models and focus on the domestic effects and transmission channels of external shocks on business cycles and financial markets. Another literature related to these works analyzes the interdependence and comovement of emerging market economy fundamentals and contagion of crises (see among others Kenourgios $\&$ Padhi (2012)), but most authors such as Li et al. (2016) focus more on how financial markets are affected and less on the interdependence of policy uncertainty.

Another important topic that has gained relevance since the GFC is the need for appropriate models to capture complex dependence structures of several variables and accurately assess risk. In one of the early contributions, Mandelbrot (1963) documented that asset returns exhibit fat tails and don't necessarily follow Gaussian distributions. It is possible to explore such dependence using non-Gaussian models with the availability of large samples of multivariate data. One such model is based on the theorem by Sklar (1959), which allows for constructing general multivariate distributions from copulas and marginal distributions. An important advantage of copula models over other dependency models is that while other models typically don't allow for asymmetric tail dependencies, copula models allow for upper, lower, symmetric and asymmetric tail dependencies. Hence, they qualify for the analysis of dependency between a large class of assets and indexes.

In this paper we focus on time constant co-dependencies between economic policy uncertainty (henceforth EPU) using C-Vine and R-Vine measures in the context of modeling the multivariate co-dependencies of four major Latin American countries (Brazil, Chile, Colombia and Mexico). Copula models are increasingly used to analyze interdependence of financial variables, especially when non-Gaussian distributions are considered (see Aas 
(2016) for a survey of financial applications of copula models).

Using the EPU measure (introduced by Baker et al. (2016)), which is based on news coverage of economic issues in respective countries, we analyze EPU linkages for the period 1996-2018. To explore possible differences after the GFC, we analyze the pre-GFC and post-GFC periods and the full sample period. Our findings indicate that there are indeed strong correlations between the EPU indexes considered and that there are tail dependencies, mostly in the pre-GFC period. We also find symmetric tail dependencies are not present in our period of analysis.

With the help of a study of this kind we extend the debate on the 2008 GFC using vine copula by examining the policy uncertainty co-dependencies among the sample nations. The present work is divided into five sections: the following section provides an overview of the methodology featuring the C-Vine and R-Vines, section 3 describes the data, section 4 presents the results, and section 5 concludes.

\section{Methodology}

There has been substantial progress in financial time series modeling ever since the seminal work of Engle (1982) with the development of the Autoregressive Conditional Heteroscedasticity $(\mathrm{ARCH})$ models for the variance to forecast changes in the volatility of financial time series. The GARCH models devised by Bollerslev (1986) allow for the use of fat tail distributions and the time-varying variances of financial returns. Bauwens et al. (2006) extend GARCH models beyond univariate specifications. Multivariate DCC GARCH models of Engle ${ }^{3}$ Sheppard (2001) and Engle (2002) nevertheless use only covariances for dependence modeling among time series, while maintaining positive definite covariance matrices. Such issues are addressed by copula modeling.

Sklar (1959) introduced the copula concept, which describes the dependence structure between variables. Copulas can be described as functions that connect multivariate and the marginal distribution functions of any dimension by utilizing all relevant information about the dependence structure between the variables that are considered to be random. Thus, copula modeling is one of the few methods that allows for greater flexibility in analyzing multivariate and marginal distributions. Earlier forms of copulas are bivariate in nature (parametric and non-parametric). However, complexity arises while modeling more than two dimensions which was later resolved by Joe (1997) with the formulation of multivariate dimension based on pair-wise copulas and Bedford $E^{3}$ Cooke (2001) using graphical measures to decompose multivariate copulas to bivariate pair-copulas.

The copula functions are technically assumed to have a vector of $Z$ random variables with marginal distribution functions of $F_{i}\left(Z_{i}\right)$ where $i=1,2, \ldots, p$. Given a set of transformation $V_{i}=F_{i}\left(Z_{i}\right)$ comprising uniformly distributed and dependent vector of random variables $V=\left(V_{1}, \ldots, V_{p}\right)$ on $[0,1]^{p}$. If if the functions of $F_{i}\left(Z_{i}\right)$ are continuous then the joint distribution function of $Z$ is assumed as:

$$
F(z)=C\left(F_{1}\left(z_{i}\right), \ldots, F_{n}\left(z_{p}\right)\right)=C\left(V_{1}, \ldots, V_{p}\right)
$$

where $C(V)$ is expressed as the copula of the distribution, $C:[0,1]^{p} \rightarrow[0,1]$ and $V=\left(V_{1}, \ldots, V_{p}\right)$. The copula $C$ can be related to a joint distribution function with vector $V$. Equation 1 is Sklar's theorem and can be extended using the copula distribution $C(V)$ as:

$$
C(v)=F\left(F_{1}^{-1}\left(v_{1}\right), \ldots, F_{p}^{-1}\left(v_{n}\right)\right)
$$

While the associated copula density can be presented as:

$$
c(v)=\frac{\partial^{p} C\left(V_{1}, \ldots, U_{p}\right)}{\partial\left(V_{1}, \ldots, V_{p}\right)}
$$


Lebrun $\mathscr{E}$ Dutfoy (2009b) explain that the joint probability density function of $Z$, which is $f_{Z}\left(z_{p}\right)=f_{z}\left(z_{1}, \ldots, z_{p}\right)$ can be is expressed as:

$$
f_{z}\left(z_{1}, \ldots, z_{p}\right)=c\left\{F_{1}\left(z_{1}\right), \ldots, F_{p}(z)\right\} \prod_{i=1}^{p} f\left(z_{1}\right)
$$

where $f_{i}\left(z_{i}\right)$ is denoted as the marginal probability density function of $z_{i}$. Equation (4) joins the copula density functions and the marginal distributions comprising all relevant information about the dependence structure of the random variables. Lebrun $\& 5$ Dutfoy (2009a) and Genest et al. (2009) computed the conditional marginal distributions of the vector of $Z$ random variables as:

$$
F_{i \mid 1, \ldots, j-1}\left(z_{i} \mid z_{i}, \ldots, z_{i-1}\right)=C_{i \mid 1, \ldots i-1}\left(v_{i} \mid v_{i}, \ldots, v_{i-1}\right)
$$

where

$$
C_{i \mid 1, \ldots, i-1}\left(v_{i} \mid v_{i}, \ldots, v_{i-1}\right)=\frac{\frac{\partial^{i-1} C\left(V_{1}, \ldots, V_{i}, 1, \ldots, 1\right)}{\partial\left(V_{1}, \ldots, V_{i-1}\right)}}{\frac{\partial^{i-1} C\left(V_{1}, \ldots, V_{i}, V_{i-1}, 1, \ldots, 1\right)}{\partial\left(V_{1}, \ldots, V_{i-1}\right)}}
$$

Considering a bivariate case, equation (6) with $v_{1}=u$ and $v_{2}=u$ can be represented as:

$$
F_{Z 2 \mid Z 1}\left(z_{2} \mid z_{1}\right)=C(u \mid v)=\frac{\partial C(v, u)}{\partial v}
$$

\section{Vine-Copulas}

Vines are graphical structures which embody joint probability distributions whereas copulas are the combined distribution of two or more random variables. The vine copula approach was pioneered by Joe (1997) and then extended by Bedford \& Cooke (2001) and Bedford $\& 3$ Cooke (2002). A vine copula is based on pair copula construction (PCC) method which includes the construction of multivariate combined distribution from bivariate and conditional bivariate copulas. Additionally, Aas et al. (2009) lay out that the paircopula construction (PCC) principle can be used with arbitrary pair-copulas, referred to as the graphical structure of R-vines. Furthermore, Dissmann et al. (2013) developed an automated algorithm of jointly searching for an appropriate R-vines tree structures, the pair-copula families and their parameters.

Along with pioneering pair-copula modeling Bedford \& Cooke (2002) also introduced socalled canonical (C-vines) and drawable vines (D-vines). These models were later extended by Aas et al. (2009) who employed non-Gaussian pair copula modeling such as the bivariate Student-t copula, bivariate Clayton copula, and bivariate Gumbel copula. C-vines exhibits star shape structures having a tree sequence, whereas, D-vines possess path structures, i.e., any particular node is not associated to more than two different nodes. For a technical description of pair-copula construction, we refer the reader to Aas et al. (2009).

Earlier versions of pair-copulas were criticized because of their inability to solve complicated models. Later, regular vine copulas (R-Vine) employing diagram algorithms as a possible way to solve the complicated models were developed. R-Vine emerged as a methodological advancement over the former because of its flexibility nature as it involves the specification of arbitrary bivariate copulas, and has the potential ability to model wide range of complicated dependencies. However, the greatest shortcomings of R-vine is the presence of curse of dimensionality. Therefore, the computational effort needed to evaluate the model increases exponentially with the dimension.

Brechmann (2010) discusses the use of simplified truncated modeling techniques to mitigate the problem of curse of dimensionality. For instance, Allen et al. (2017) advise truncation of a regular vine at level $M$ where independent copulas replace any pair-copulas that 
are either equal to or larger than $M$. These independent copulas are known as Gaussian copulas, which are easier in specification among other variants of copulas and are simpler in their interpretation of the correlation parameter.

\section{Data}

We use EPU index data for four major Latin American countries, Mexico, Brazil, Chile and Colombia as compiled by Baker et al. (2016). The index is based on news coverage frequency of policy-related economic issues and serves as a proxy for economic uncertainty. There are many uncertainty measures such as the VIX for developed countries. In contrast, uncertainty measures are scant for emerging economies and the EPU index provides a scaled measure of the appearance of uncertainty in news surrounding economic issues. The data spans the period from January 1996 to May 2018. The advantage of using monthly data is that extreme co-movement within the sample period is expected more frequently and potential tail dependencies can be captured more accurately. We also believe that the movements in the economic policy uncertainty indices among the nations can reflect the situation of the intra economic and market conditions to a large extent. We divide our period of analysis into the pre-GFC (January 1996-December 2008), post-GFC (January 2009-December 2018) periods, and the full sample baseline period (January 1996-December 2018).

\section{Results}

As explained above, we make use of C-Vine and R-Vine copulas. The use of C-Vines is appropriate when there are pivotal elements within the selection, i.e. when the dependence structure of the other variables is measured with respect to the pivotal variable. The pivotal variable is the one that maximizes the sum of Kendall's tau, i.e. pairwise dependencies. R-Vines on the other hand are less restrictive and don't necessarily need pivotal elements. We use these two models to test and see whether the EPU index of a country plays a pivotal role in the dependency structure of country combinations. In the following, we present the results for the $\mathrm{C}$-Vine and $\mathrm{R}$-Vine copulas for the periods under consideration.

\subsection{C-Vine Copulas}

We present the results of the dependence structure of the EPU indices of the four different nations using the $\mathrm{C}$-Vine and R-Vine co-dependency models. These models would help to trace the dependencies between uncertainty indices which plays a vital role in the underpinning of economies trade cycles. To consider the possibility of tail dependencies, we allow for six copula families; Gaussian/Normal copula, Student t copula, Clayton copula, Gumbel copula, Frank copula and Joe copula. Details on the characteristics of these copula families can be found in Joe (2014). In summary, Gaussian and Frank copula don't exhibit tail dependencies, Gumbel and Joe copula exhibit upper tail dependencies and Clayton copula exhibit lower tail dependencies. The t copula stands out as it exhibits both upper and lower tail dependency.

An advancement is the successful application of the C-vines in the modeling of such dependency patterns (Czado et al. (2013)). The time series data of the four countries EPU indices are presented in figure 1 . We divide the analysis into three sub-periods covering the pre-GFC, the post-GFC, and the full sample periods and further document the results of the three sub sample analysis in section 4 .

\section{C-Vine: Pre-GFC Period}

Most Latin America economies recorded substantial economic growth between 2003 and 2007. Past studies discuss four important factors held responsible for this boom: growing 
Figure 1: EPU Index (in logs)

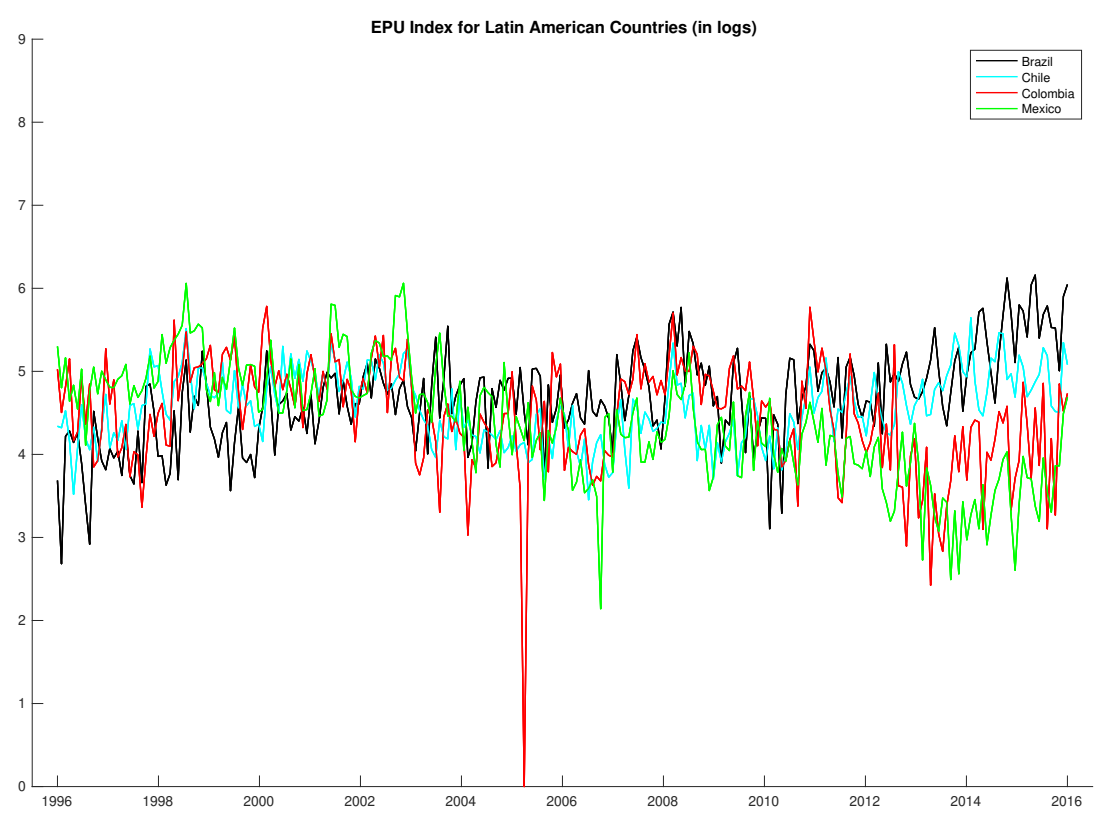

international trade (9.3\% per year between 2003-06), a significant increase in commodity prices, high levels of remittances, and remarkable financing conditions (Ocampo (2009)). Given this background, to map the Pre-GFC period, we show the structure of C-Vines which is selected, based on a root node that maximizes the sum of pairwise dependencies to this node (see figure 2).

Tree 1 in figure 2 shows that the EPU index of Chile is the pivotal variable, linking all the EPU of the other countries. We employ AIC to select the appropriate copula which is "best fit" for every pair of variables from the following: $1=$ Gaussian/Normal copula, $2=$ Student t copula (t-copula), $3=$ Clayton copula, $4=$ Gumbel copula and survival Gumbel Copula, $5=$ Frank copula, $6=$ Joe copula. The AIC adjusts the log likelihood of a copula for the number of estimated parameters (Dissmann et al. (2013)). We further calculate the transformed observations from the pair copulas which enter as input parameters for the next trees (see tree 2). Similarly, the transformed observations are obtained by using the C-Vine proximity conditions principles, and finding a maximum dependence tree for the period 2 as shown in tree 2 of figure 2. As an additional measure of dependency, we use Kendall's rank correlation coefficient (tau).

Table 1: C-Vine Copula Structure

\begin{tabular}{ccccc}
\hline & Brazil & Chile & Colombia & Mexico \\
\hline Brazil & 3 & 0 & 0 & 0 \\
Chile & 4 & 1 & 0 & 0 \\
Colombia & 1 & 4 & 2 & 0 \\
Mexico & 2 & 2 & 4 & 4 \\
\hline
\end{tabular}


Figure 2: Results C-Vine Tree-1 and 2, Pre-GFC

Tree 1

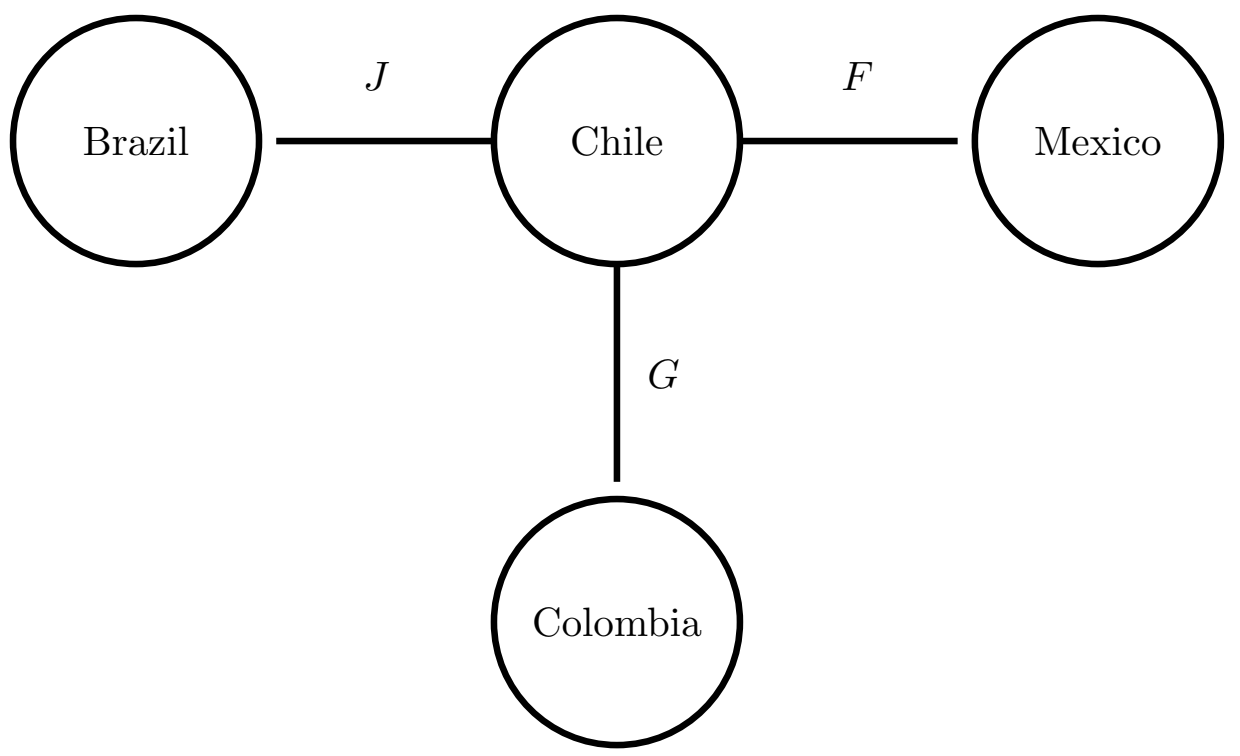

Tree 2

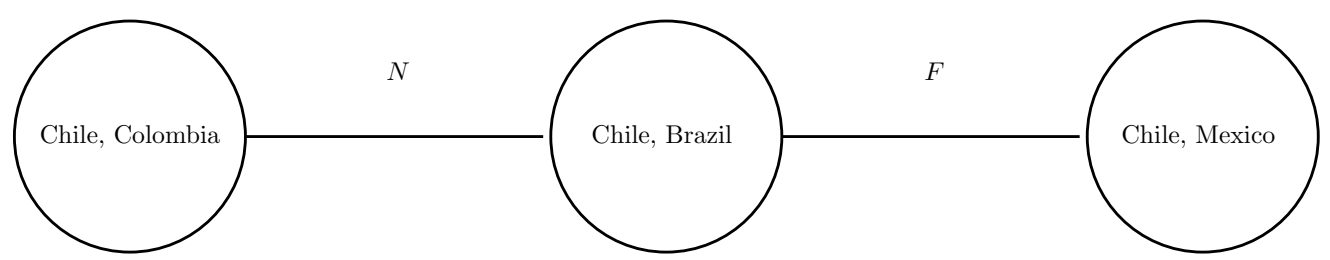

Table 2: C-Vine Copula Specification Matrix

\begin{tabular}{ccccc}
\hline & Brazil & Chile & Colombia & Mexico \\
\hline Brazil & 0 & 0 & 0 & 0 \\
Chile & 3 & 0 & 0 & 0 \\
Colombia & 1 & 5 & 0 & 0 \\
Mexico & 4 & 6 & 5 & 0 \\
\hline
\end{tabular}

Table 3: C-Vine Copula Tau Matrix

\begin{tabular}{ccccc}
\hline & Brazil & Chile & Colombia & Mexico \\
\hline Brazil & 0 & 0 & 0 & 0 \\
Chile & 0.114 & 0 & 0 & 0 \\
Colombia & 0.112 & -0.147 & 0 & 0 \\
Mexico & 0.331 & 0.110 & 0.347 & 0 \\
\hline
\end{tabular}

In table 1, we observe that during the pre-GFC period, the strongest individual correlations are between Brazil which is situated in the first column, and Mexico located in the final row, while other individual diagonal entries define the edges. Brazil is correlated with Columbia, followed by its relationship with Chile. C-Vines are less flexible as compared to $\mathrm{R}$-Vines structure because in case of C-Vines the same number usually appear across the 
rows. In a nutshell, the number frequently appear in the nodes at that level in the tree.

Table 2 presents and confirms the choice of the appropriate copula that is used to capture the dependencies between several pairs of countries EPU indices. In table 2, it is clear from the bottom of column 1 that number 4 copula (the Gumbel copula) is applied, to capture the dependency between Brazil and Mexico, and followed by the use of Gaussian copula (1) showing the relationship between Brazil and Colombia, and so forth. We also find that table 2 has all the six categories of copula except Student t copula. The Frank copula appears twice in the table, followed by the Gaussian copula, the Gumbel copula, the Clayton copula and finally the Joe copula that appear once each. In short, these results imply that in half of the results, there are no tail dependencies. For the rest, upper tail dependencies appear more commonly than lower tail dependencies.

We report the tau matrix for the C-Vine copulas during the pre-GFC period in table 3. A positive value of tau coefficient signifies positive and symmetric effects among the economies under consideration. Similarly, a positive value of the tau coefficient also implies that a negative change in the policy uncertainty will bring a similar (negative) change in the policy uncertainty of the other dependent country. We find that the last row of table 3 captures the positive and strongest dependencies between the countries EPU indices. Colombia and Mexico are reported to have the strongest positive dependencies between any combinations of countries EPU indices, whereas our findings show imply dependencies between Chile and Colombia in the pre-GFC period.

\section{C-Vine: Post-GFC Period}

We now present the results of the C-Vines structure for the Post-GFC period. In figure 3 , we show the first tree and the second tree of the post-GFC period.

Figure 3: Results C-Vine Tree-1 and 2, Post-GFC

Tree 1

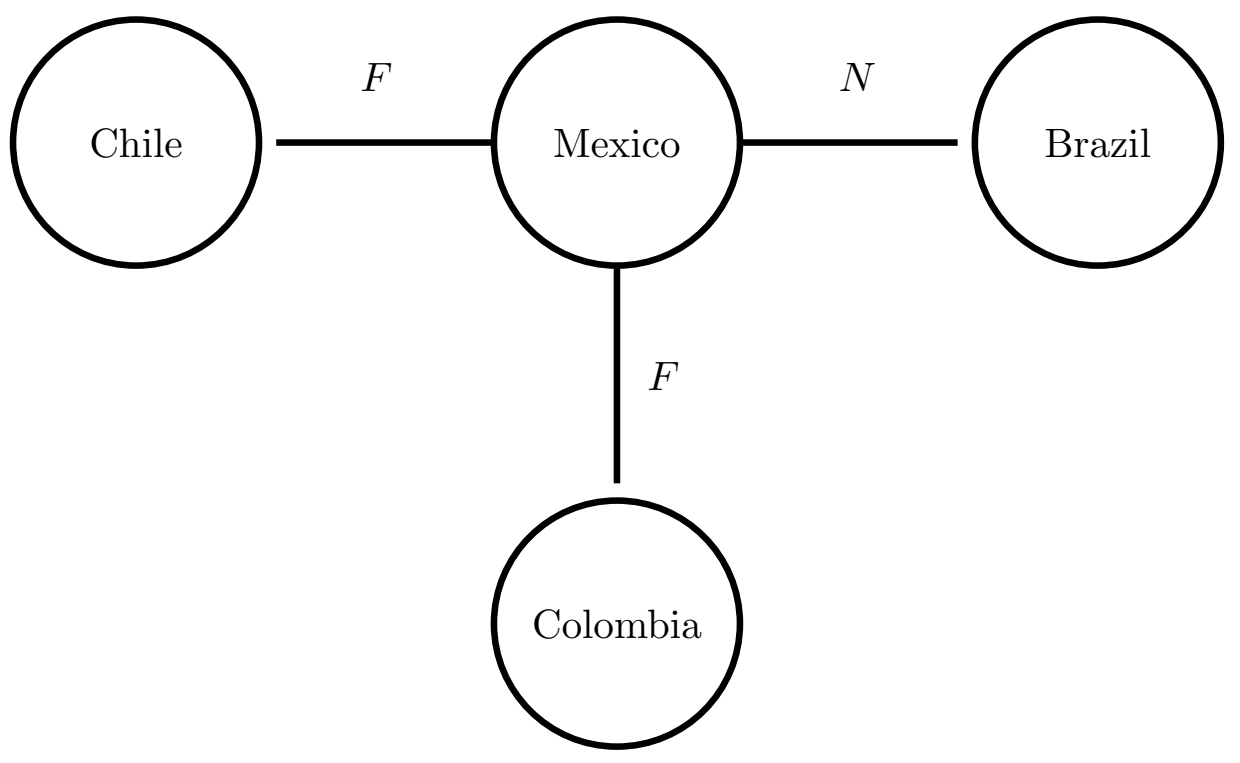

We then present the post-GFC C-Vine copula structure in table 4, and the post-GFC C-Vine Copula Specification Matrix in table 5. The top and bottom of the first column reveal the strongest correlations between Brazil and Mexico. 
Tree 2

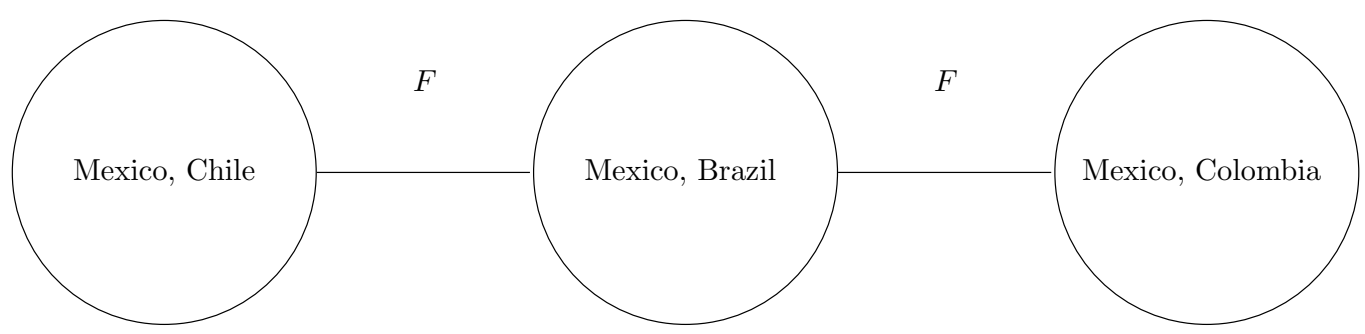

Table 4: C-Vine Copula Structure

\begin{tabular}{ccccc}
\hline & Brazil & Chile & Colombia & Mexico \\
\hline Brazil & 2 & 0 & 0 & 0 \\
Chile & 3 & 1 & 0 & 0 \\
Colombia & 1 & 3 & 3 & 0 \\
Mexico & 4 & 4 & 4 & 4 \\
\hline
\end{tabular}

Table 5: C-Vine Copula Specification Matrix

\begin{tabular}{ccccc}
\hline & Brazil & Chile & Colombia & Mexico \\
\hline Brazil & 0 & 0 & 0 & 0 \\
Chile & 5 & 0 & 0 & 0 \\
Colombia & 5 & 5 & 0 & 0 \\
Mexico & 5 & 1 & 5 & 0 \\
\hline
\end{tabular}

Table 6: C-Vine Copula Tau Matrix

\begin{tabular}{ccccc}
\hline & Brazil & Chile & Colombia & Mexico \\
\hline Brazil & 0 & 0 & 0 & 0 \\
Chile & 0.012 & 0 & 0 & 0 \\
Colombia & 0.276 & 0.139 & 0 & 0 \\
Mexico & -0.147 & -0.131 & 0.398 & 0 \\
\hline
\end{tabular}

From table 5, we find a change in the type of copula for capturing the dependencies in the post-GFC period. The Frank copula appears five times in the post-GFC period, while the use of the Gaussian copula during the pre-GFC period has remained constant as compared to the post-GFC period. During the post-GFC period, we did not find any evidence of the application of other copula families that exhibit tail dependencies. The appearance of Gaussian copula and Frank copula and the absence of the Student t copula suggests that tail dependencies are less important in the post-GFC period in comparison to the pre-GFC period.

The tau matrix of the post-GFC period is presented in table 6 . We observe a complex structure of dependencies between the countries' EPU indices when compared to those of the post-GFC period. There is also an increase in negative dependencies: while there was one negative tau coefficient in the matrix during the pre-GFC period, this number has increased to two altogether in the post-GFC period, specifically for the country combinations of Brazil and Mexico, and Chile and Mexico. Although we find a positive coefficient value of tau in the case of Brazil and Chile, implying symmetric changes in the policy uncertainty among the nations, the dependency is weakest as revealed from the low value of the tau 
coefficient. We find that any positive (negative) change in the policy uncertainty index of Colombia will affect positively (negatively) the policy uncertainty index of Mexico and imply the strongest dependency among all other combinations of the countries under consideration.

\section{C-Vine: Full Sample Period}

Figure 4: Results C-Vine Tree-1 and 2, Full Sample Period

Tree 1

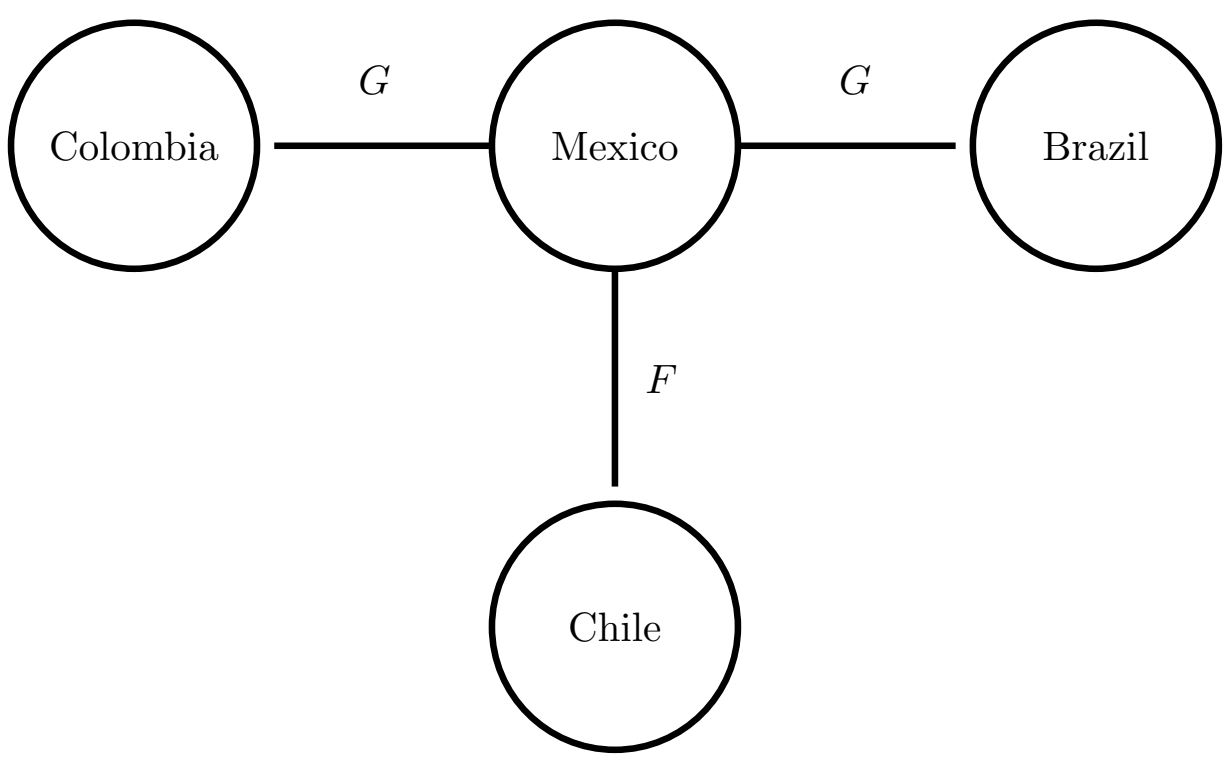

Tree 2

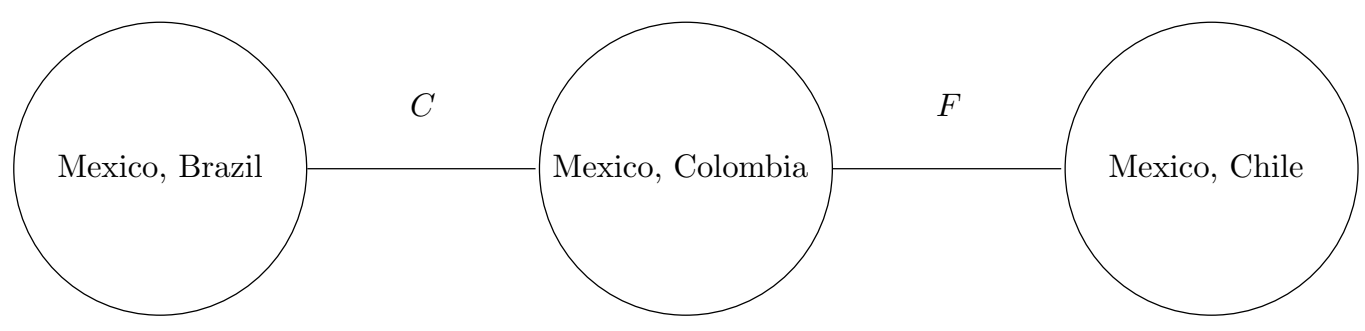

From table 7, we find that the top and bottom of the first column divulge the strongest correlations between Brazil and Mexico. Especially the value assigned to Mexico remains the same at the bottom across the last row. As we move from the bottom to the top, we find that Brazil is correlated with Colombia, and conditioned by its relationship with Chile. It is clear that the estimates of $\mathrm{C}$-Vines appears to be less flexible since the same number appears in most of the cases across the rows.

Table 8 show the range of best fitted copula. The C-Vine copula matrix presents the dependencies between different pairs of countries EPU indices. We observe from the bottom row of the column 1 that the number 4 (Gumbel) copula appears twice, followed by number 5 (Frank) copula which also appeared twice conditioned by number 3 (Clayton) copula and number 1 (Gaussian) copula which appeared once each. Similar to the pre-GFC period, we note that when taking the full period into consideration, there are no tail dependencies in half of the results and that for the rest, upper tail dependencies appear to be more relevant than lower tail dependencies. 
Table 7: C-Vine Copula Structure

\begin{tabular}{ccccc}
\hline & Brazil & Chile & Colombia & Mexico \\
\hline Brazil & 1 & 0 & 0 & 0 \\
Chile & 2 & 2 & 0 & 0 \\
Colombia & 3 & 3 & 3 & 0 \\
Mexico & 4 & 4 & 4 & 4 \\
\hline
\end{tabular}

Table 8: C-Vine Copula Specification Matrix

\begin{tabular}{ccccc}
\hline & Brazil & Chile & Colombia & Mexico \\
\hline Brazil & 0 & 0 & 0 & 0 \\
Chile & 1 & 0 & 0 & 0 \\
Colombia & 3 & 5 & 0 & 0 \\
Mexico & 4 & 5 & 4 & 0 \\
\hline
\end{tabular}

Table 9: C-Vine Copula Tau Matrix

\begin{tabular}{ccccc}
\hline & Brazil & Chile & Colombia & Mexico \\
\hline Brazil & 0 & 0 & 0 & 0 \\
Chile & 0.082 & 0 & 0 & 0 \\
Colombia & 0.108 & 0.093 & 0 & 0 \\
Mexico & 0.076 & 0.173 & 0.142 & 0 \\
\hline
\end{tabular}

The tau matrix of the full sample estimates is presented in table 9 . It is evident that there are no negative tau coefficients in the matrix during the full sample estimates, which is consistent with the pre-GFC period. The absence of negative coefficients again highlight no evidence of negative dependencies between the countries EPU indices. The combinations of all countries EPU indices denotes positive dependencies among each other. In other words, positive (negative) change in economic uncertainty of a country would bring similar positive (negative) change in the economy uncertainty of the other country. Countries namely Chile and Mexico are reported to be having the highest positive dependencies while the countries Brazil and Mexico show lowest positive dependencies in the full sample period.

From the above discussions pertaining to the $\mathrm{C}-\mathrm{Vines}$ results, it is interesting to note that Gaussian copula appears in the pre-GFC, post-GFC and full sample estimates. However, we did not find any evidence of Student $t$ copula in any of the estimates. The frequent appearance of the Gaussian copula and the absence of the Student $t$ copula imply less importance on the tails of the distributions in the pre-GFC period, the post-GFC period and the full sample estimates.

\section{$4.2 \quad$ R-Vine Copulas}

\section{R-Vine: Pre-GFC Period}

We now present the tree structure for the pre-GFC period using the R-Vine copula.

It is evident from figure 5 that the R-Vine structure is more flexible as compared to the $\mathrm{C}$-Vine structure. We find that the EPU indices of all the countries are associated to one another, except Brazil and Mexico which can also be seen in tree 2 and tree 3 . Therefore, as a pair the EPU indices of Brazil, Columbia and Chile have the strongest co-dependency and on the other hand the EPU indices of Mexico, Chile and Columbia also have strongest co-dependency among one another. In table 10, we show the appropriate fitted copulas. It 
Tree 1

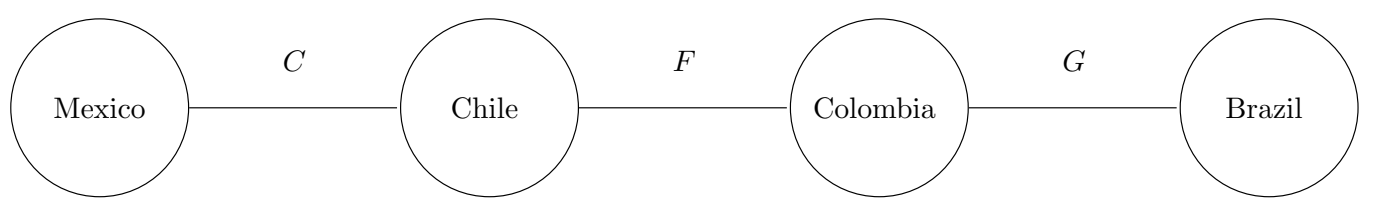

Tree 2

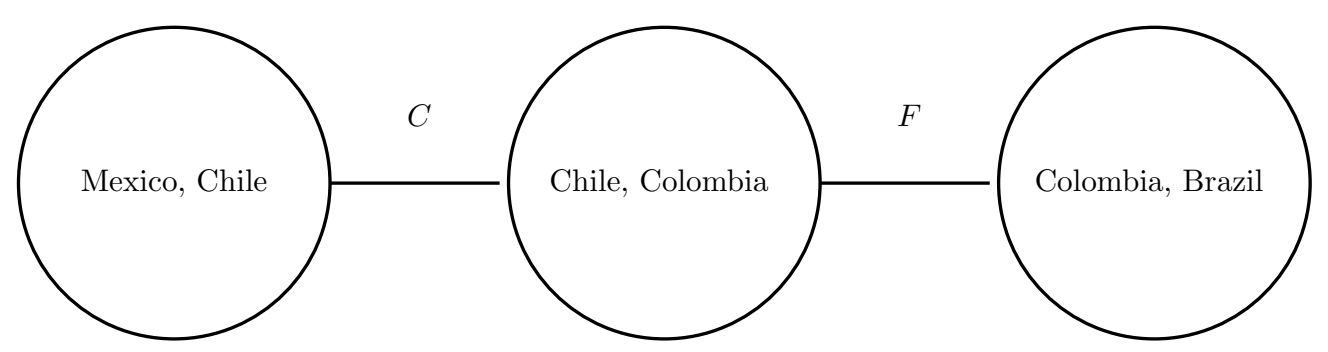

is clear from the table that different dependencies conditioned across the same node employ three different copulas. In this case, with reference to the first column, the first copula used is the Frank copula (no 5), followed by Gumbel copula (no 4) and Joe copula (no 6). The bottom row is also captured by Joe copula, followed by Gumbel copula and Frank copula. We can note that the copulas employed here are slightly different from the pre-GFC period $\mathrm{C}$-Vine results as upper tail dependencies play the most prominent role.

Table 10: R-Vine Copula Specification Matrix

\begin{tabular}{ccccc}
\hline & Brazil & Chile & Colombia & Mexico \\
\hline Brazil & 0 & 0 & 0 & 0 \\
Chile & 5 & 0 & 0 & 0 \\
Colombia & 4 & 3 & 0 & 0 \\
Mexico & 6 & 4 & 5 & 0 \\
\hline
\end{tabular}

Table 11: R-Vine Copula Tau matrix

\begin{tabular}{ccccc}
\hline & Brazil & Chile & Colombia & Mexico \\
\hline Brazil & 0 & 0 & 0 & 0 \\
Chile & -0.169 & 0 & 0 & 0 \\
Colombia & 0.021 & 0.089 & 0 & 0 \\
Mexico & 0.138 & 0.331 & 0.347 & 0 \\
\hline
\end{tabular}

We report the tau matrix for the pre-GFC period using R-Vines in table 11 . We find similar results for R-Vines when compared to C-Vines results shown in table 3 for the preGFC period. Once again, the entries in the bottom of the row indicates the positive and strongest co-dependencies among the countries EPU indices. We also find a positive coefficient of the tau for rest of the diagonals except for the country combination Brazil and Chile. This implies that a positive or negative changes in the economic policy uncertainty of Brazil would bring negative or positive changes in the policy uncertainty of Chile respectively. We document the strongest and positive dependencies between Colombia and Mexico as compared to any other countries combinations corroborating our results of C-Vines. Further, 
Tree 3

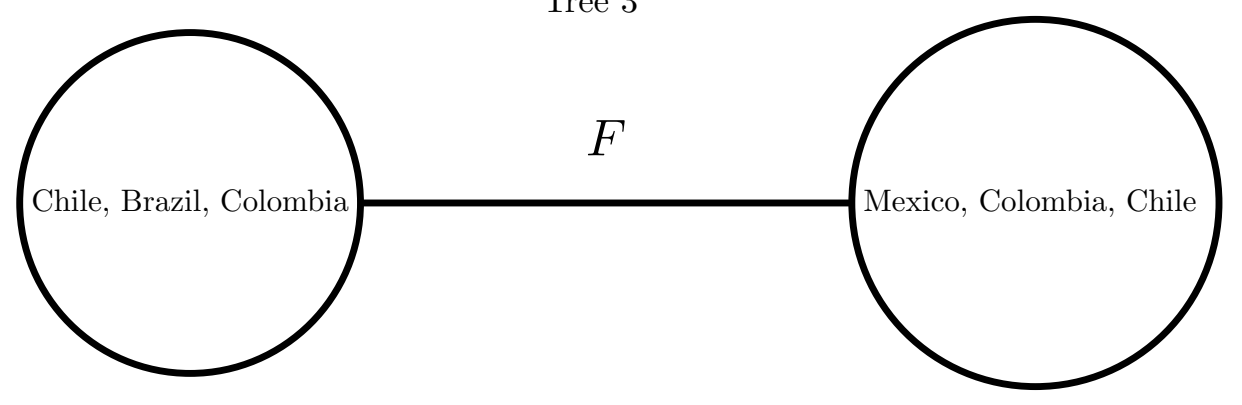

the weakest positive dependency is found for the countries Brazil and Colombia.

\section{R-Vine: Post-GFC Period}

We now focus our attention to the post-GFC period. We are able to figure out that the trees shown in the figure 6 have changed considerably as compared to the pre-GFC period. Figure 6 reveals the dependencies between the EPU indices among the sample nations included in this study. It is evident from tree 1 that Brazil is now linked to Colombia through Chile and Mexico. During the pre-GFC period, we can note that Brazil was linked to Mexico via Colombia and Chile (see figure 6).

Figure 6: Results R-Vine Tree-1, 2 and 3, Post-GFC

Tree 1

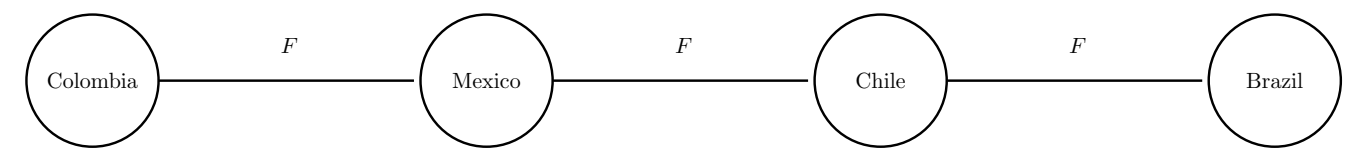

Tree 2

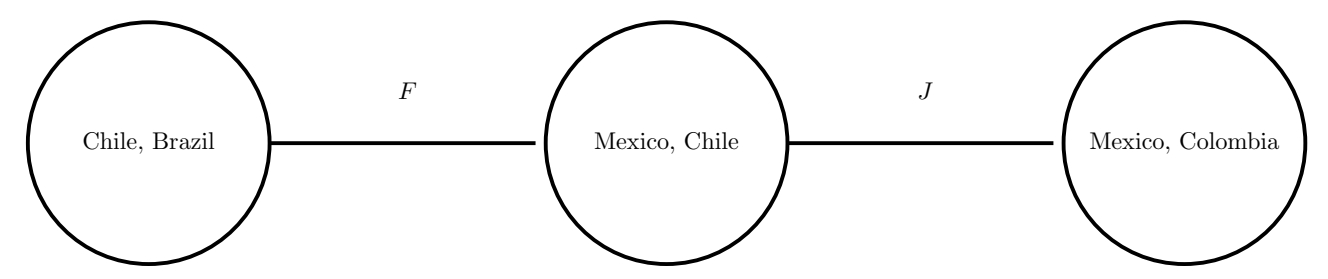

Tree 3

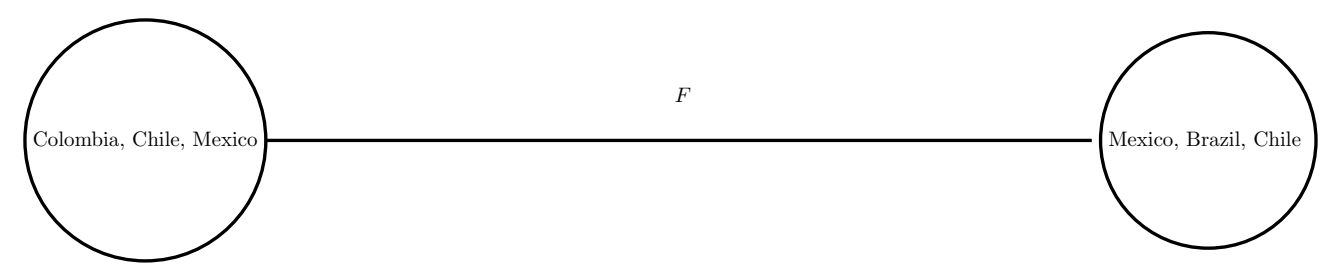

Table 12 shows the type of copulas used to map the co-dependencies among the EPU indices. We find that the Frank copula dominates with five entries while the Joe copula appears only once. Similar to the results of C-Vines, these results confirm that in the postGFC period tail dependencies become less relevant. The tau matrix is presented in table 13 
Table 12: R-Vine Copula Specification Matrix

\begin{tabular}{ccccc}
\hline & Brazil & Chile & Colombia & Mexico \\
\hline Brazil & 0 & 0 & 0 & 0 \\
Chile & 5 & 0 & 0 & 0 \\
Colombia & 5 & 6 & 0 & 0 \\
Mexico & 5 & 5 & 5 & 0 \\
\hline
\end{tabular}

Table 13: R-Vine Copula Tau matrix

\begin{tabular}{ccccc}
\hline & Brazil & Chile & Colombia & Mexico \\
\hline Brazil & 0 & 0 & 0 & 0 \\
Chile & 0.133 & 0 & 0 & 0 \\
Colombia & -0.069 & 0.061 & 0 & 0 \\
Mexico & 0.304 & -0.147 & 0.398 & 0 \\
\hline
\end{tabular}

and reveals that the dependencies between the countries have significantly changed during the post-GFC period. Interestingly, there are now two negative entries, specifically for the country combinations Chile and Mexico and Brazil and Colombia for the post-GFC period. We also find that the strongest and positive dependency is exhibited by the combination Colombia and Mexico while the weakest relationship is exhibited by Chile and Colombia. Therefore, the results for the post-GFC period are mixed and similar to the C-vines results as reported earlier in the present study.

\section{R-Vine: Full Sample Period}

The trees of the vine copula for the full sample period are presented in figure 7.

Figure 7: Results R-Vine Tree-1, 2 and 3, Full Sample Period

Tree 1

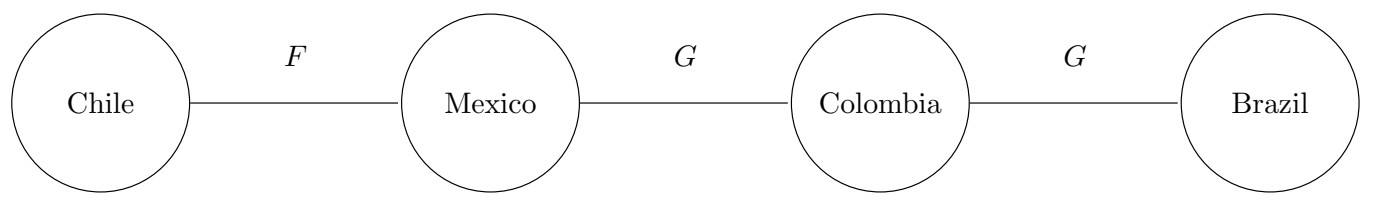

Tree 2

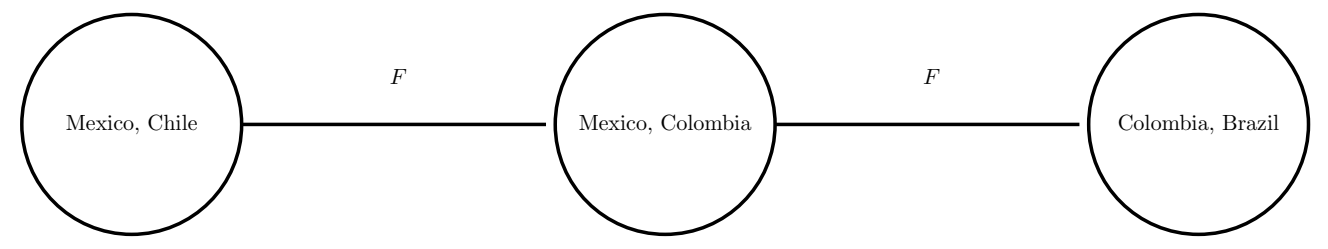

Tree 3

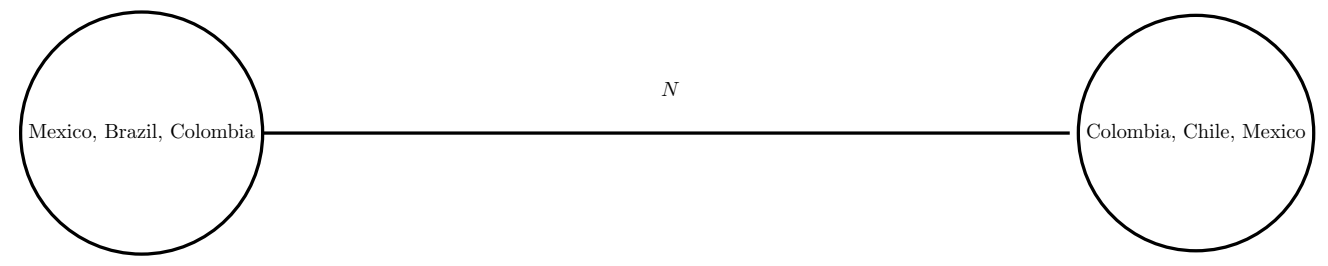


It is again clear from the R-Vine copula structure estimates that these estimates are more flexible relative to the $\mathrm{C}$-Vine structure. The trees exhibit strong codependencies between Mexico, Brazil and Colombia on one hand and Colombia, Chile and Mexico on the other hand, as is also apparent in tables 14 and 15 .

In table 15, we show the type of best fit copula for the present analysis. It can be seen that different copulas are used, conditioned across the same node implying divergent dependencies among the countries EPU indices. We notice that Frank copula appears the maximum time followed by Clayton, Gumbel and Gaussian copula with one entry each. Departing from previous studies (Albulescu et al. (2018); Allen et al. (2017)), we did not find any evidence of Student t copula, highlighting diminished importance of fat tails in any of the estimates followed by pre-GFC, post-GFC and full sample period which is also similar for the $\mathrm{C}$-Vines results.

Table 14: R-Vine Copula Structure

\begin{tabular}{ccccc}
\hline & Brazil & Chile & Colombia & Mexico \\
\hline Brazil & 1 & 0 & 0 & 0 \\
Chile & 2 & 2 & 0 & 0 \\
Colombia & 4 & 3 & 3 & 0 \\
Mexico & 3 & 4 & 4 & 4 \\
\hline
\end{tabular}

Table 15: R-Vine Copula Specification Matrix

\begin{tabular}{ccccc}
\hline & Brazil & Chile & Colombia & Mexico \\
\hline Brazil & 0 & 0 & 0 & 0 \\
Chile & 1 & 0 & 0 & 0 \\
Colombia & 5 & 5 & 0 & 0 \\
Mexico & 3 & 5 & 4 & 0 \\
\hline
\end{tabular}

Table 16: R-Vine Copula Tau matrix

\begin{tabular}{ccccc}
\hline & Brazil & Chile & Colombia & Mexico \\
\hline Brazil & 0 & 0 & 0 & 0 \\
Chile & 0.085 & 0 & 0 & 0 \\
Colombia & 0.059 & 0.093 & 0 & 0 \\
Mexico & 0.114 & 0.173 & 0.142 & 0 \\
\hline
\end{tabular}

From table 16, it is clear that the dependencies between the countries EPU indices is strong and positive. The results of the full sample estimates are similar to the earlier findings in this study for C-Vines copula (see table 9). Further, we also note that the strongest and positive dependency is between Chile and Mexico while the weakest but positive dependency is documented for Brazil and Colombia.

The application of C-Vine and R-Vine copulas in the present study helps in capturing the dependency between the EPU indices of Brazil, Chile, Colombia and Mexico. The increase in the economic integration in terms of cross border trade and capital flows stirs the dependencies among these Latin American countries. We trace the dependencies among the nations determined by their EPU indices during different time periods. About two-fifths of emerging markets' total trade is accounted for having trade with other emerging markets, which is almost double presently as compared to two decades ago (Kose et al. (2008)). Past studies such as the dividend discount model, project that the effect of any uncertainty (say GFC in this case) on the real economy would immediately affect the stock returns and several other factors related to the financial market (Gordon $\& 5$ Shapiro (1956)). 
Similarly, there is also a possibility of effects of enduring uncertainty percolating from one economy to the other. Therefore, the economic uncertainty in one economy may penetrate the other economies through contagion. The interdependencies between the EPU indices between the nations in our study explain the persistence of contagion that differs from the pre-GFC and post-GFC periods evident through differences in the copula structure. The present research shows that the economies investigated are closely interlinked, dissecting the decoupling debate about the economies' uncertainty conditions.

\section{Conclusion}

As argued in Ozturk $\mathscr{E}$ Sheng (2018), the post-GFC period is marked by heightened uncertainty in trade, monetary policy and financial markets. Researchers in economics and finance pay more attention to the reasons behind financial crises and quantify interrelated economic phenomena, using various dependent parameters. In this context, we investigated the co-dependencies between the EPU indices of four Latin American countries. To achieve that, we employed a simple vine copula framework. There are several important results: in both the C-Vine structure and R-Vine structures, the rank correlation coefficient (tau) matrices show that the dependency among the EPU indices is mostly positive for the four countries considered, implying similar changes in economic policy uncertainty among the nations under consideration for the full sample period. For the pre-GFC and post-GFC period however, we find mixed signs for the countries' EPU indices. Especially for country combinations involving Chile, there are multiple cases where the coefficient is negative, implying opposite movement of the EPU index.

Another important finding relates to the absence of Student $t$ copula in the present study in all time periods considered, including financial turmoil, which highlights that symmetric tail dependencies are absent. Further, asymmetric tail dependencies, mostly in the form of upper tail dependency, are present when the pre-GFC period or the full period are considered. In contrast, there are no tail dependencies when the post-GFC period is considered. This may imply that after the global financial crisis of 2008, there was a decoupling in economic policy uncertainty among the countries considered and that as a result tail dependencies became less relevant.

Our results carry relevance for the analysis of macroeconomic and financial topics such as business cycles, stock markets and policy coordination.

\section{References}

AAs, K. 2016. Pair-copula constructions for financial applications: A review. Econometrics, 4(4):43.

Aas, K., Czado, C., Frigessi, A. \& Bakken, H. 2009. Pair-copula constructions of multiple dependence. Insurance: Mathematics and economics, 44(2):182-198.

Albulescu, C.T., Aubin, C., Goyeau, D. E Timari, A.K. 2018. Extreme co-movements and dependencies among major international exchange rates: A copula approach. The Quarterly Review of Economics and Finance.

Allen, D.E., McAleer, M. E Singh, A.K. 2017. Risk measurement and risk modelling using applications of vine copulas. Sustainability, 9(10):1762.

BAker, S.R., Bloom, N. \& DAVIS, S.J. 2016. Measuring economic policy uncertainty. The Quarterly Journal of Economics, 131(4):1593-1636.

Bauwens, L., Laurent, S. E R Rombouts, J.V. 2006. Multivariate garch models: a survey. Journal of applied econometrics, 21(1):79-109.

Bedford, T. E Cooke, R.M. 2001. Probability density decomposition for conditionally dependent random variables modeled by vines. Annals of Mathematics and Artificial intelligence, 32(14):245-268.

BedFord, T. E CoOKe, R.M. 2002. Vines: A new graphical model for dependent random variables. Annals of Statistics, pages 1031-1068. 
Biljanovska, N., Grigoli, F. $\mathscr{E}$ Hengge, M. 2017. Fear thy neighbor: Spillovers from economic policy uncertainty. International Monetary Fund.

Bloom, N. 2009. The impact of uncertainty shocks. econometrica, 77(3):623-685.

Bollerslev, T. 1986. Generalized autoregressive conditional heteroskedasticity. Journal of econometrics, 31(3):307-327.

Brechmann, E. 2010. Truncated and simplified regular vines and their applications. Thesis.

Carrière-Swallow, Y. ES CÉspedes, L.F. 2013. The impact of uncertainty shocks in emerging economies. Journal of International Economics, 90(2):316-325.

Chan-Lau, M.J.A. 2010. The Global Financial Crisis and its Impact on the Chilean Banking System. 10-108. International Monetary Fund.

Colombo, V. 2013. Economic policy uncertainty in the us: Does it matter for the euro area? Economics Letters, 121(1):39-42.

Czado, C., Brechmann, E.C. \& Gruber, L. 2013. Selection of vine copulas. In Copulae in Mathematical and Quantitative Finance, pages 17-37. Springer.

Dissmann, J., Brechmann, E.C., Czado, C. E K Kurowicka, D. 2013. Selecting and estimating regular vine copulae and application to financial returns. Computational Statistics $\&$ Data Analysis, 59:52-69.

Engle, R. 2002. Dynamic conditional correlation: A simple class of multivariate generalized autoregressive conditional heteroskedasticity models. Journal of Business 85 Economic Statistics, 20(3):339-350.

ENGLE, R.F. 1982. Autoregressive conditional heteroscedasticity with estimates of the variance of united kingdom inflation. Econometrica: Journal of the Econometric Society, pages 987-1007.

Engle, R.F. \& S ShepPARD, K. 2001. Theoretical and empirical properties of dynamic conditional correlation multivariate garch. Technical Report, National Bureau of Economic Research.

Fernández-Villaverde, J., Guerrón-Quintana, P., Rubio-Ramírez, J.F. $\&$ Uribe, M. 2011. Risk matters: The real effects of volatility shocks. American Economic Review, 101(6):2530-61.

Genest, C., RÉmillard, B. $\&$ Beaudoin, D. 2009. Goodness-of-fit tests for copulas: A review and a power study. Insurance: Mathematics and economics, 44(2):199-213.

Gordon, M.J. \& Shapiro, E. 1956. Capital equipment analysis: the required rate of profit. Management science, 3(1):102-110.

JoE, H. 1997. Multivariate models and multivariate dependence concepts. Chapman and Hall/CRC.

JoE, H. 2014. Dependence modeling with copulas. Chapman and Hall/CRC.

Kenourgios, D. \& PAdHi, P. 2012. Emerging markets and financial crises: regional, global or isolated shocks? Journal of Multinational Financial Management, 22(1-2):24-38.

KLÖSSNER, S. \& S SEKKEL, R. 2014. International spillovers of policy uncertainty. Economics Letters, 124(3):508-512.

Kose, M.A., Otrok, C. $\&$ Prasad, E.S. 2008. Global business cycles: convergence or decoupling? Technical Report, National Bureau of Economic Research.

Lebrun, R. ES Dutfoy, A. 2009a. Do rosenblatt and nataf isoprobabilistic transformations really differ? Probabilistic Engineering Mechanics, 24(4):577-584.

Lebrun, R. \& Dutfoy, A. 2009b. An innovating analysis of the nataf transformation from the copula viewpoint. Probabilistic Engineering Mechanics, 24(3):312-320.

Li, X.-l., Balcilar, M., Gupta, R. EG Chang, T. 2016. The causal relationship between economic policy uncertainty and stock returns in china and india: evidence from a bootstrap rolling window approach. Emerging Markets Finance and Trade, 52(3):674-689.

Mandelbrot, B. 1963. New methods in statistical economics. Journal of political economy, 71(5):421-440. 
OCAMPO, J.A. 2009. Latin america and the global financial crisis. Cambridge journal of economics, $33(4): 703-724$.

Ozturk, E.O. \& S Sheng, X.S. 2018. Measuring global and country-specific uncertainty. Journal of International Money and Finance, 88:276-295.

Sklar, M. 1959. Fonctions de repartition an dimensions et leurs marges. Publ. inst. statist. univ. Paris, 8:229-231.

Stiglitz, J.E. 2010. The Stiglitz Report: Reforming the international monetary and financial systems in the wake of the global crisis. The New Press. 